\title{
PREOPERATIVE ULTRASONOGRAPHIC DETERMINATION OF DEEP SURGICAL MARGIN IN SQUAMOUS CELL CARCINOMA OF BUCCAL MUCOSA
}

\author{
Cecil T. Thomas ${ }^{1}$, Sramana Mukhopadhyay², Aparna Irodi' ${ }^{3}$ Betty Simon ${ }^{4}$, Amit J. Tirkey5, Rajinikanth J6 John C. Muthusami ${ }^{7}$, \\ Pranay Gaikwad ${ }^{8}$
}

\begin{abstract}
${ }^{1}$ Associate Professor, Department of General Surgery Unit 1-Head and Neck Oncosurgery, Christian Medical College, Vellore, Tamilnadu, India.

${ }_{2}^{2}$ Associate Professor, Department of Pathology, Christian Medical College, Vellore, Tamilnadu, India.

3 Professor, Department of Radiodiagnosis, Christian Medical College, Vellore, Tamilnadu, India.

${ }^{4}$ Professor, Department of Radiodiagnosis, Christian Medical College, Vellore, Tamilnadu, India.

5 Professor, Department of General Surgery Unit 1-Head and Neck Oncosurgery, Christian Medical College, Vellore, Tamilnadu, India. ${ }^{6}$ Professor, Department of General Surgery Unit 1-Head and Neck Oncosurgery, Christian Medical College, Vellore, Tamilnadu, India.

7 Professor, Department of General Surgery Unit 1-Head and Neck Oncosurgery, Christian Medical College, Vellore, Tamilnadu, India. ¿Professor, Department of General Surgery Unit 1-Head and Neck Oncosurgery, Christian Medical College, Vellore, Tamilnadu, India.
\end{abstract} ABSTRACT

\section{BACKGROUND}

Surgical margins are a modifiable factor in the management of buccal cancers. The deep margin is often compromised in favour of aesthetics of a preserved skin flap over the cheek. We wanted to study the impact of deep margin assessment by ultrasound, subsequent surgical clearance, and local recurrence rates at 2 years of follow up in carcinoma of buccal mucosa.

\section{METHODS}

This was a combined prospective and retrospective single institution study of patients with buccal carcinoma assessed by ultrasound and clinical examination, conducted to test the histopathological adequacy, the need for reconstructive procedures and for adjuvant therapy, in 15 patients with the buccal squamous cell carcinoma by sonographic determination of tumour-skin distance. The findings were compared with those from the historical controls. In the study group, the overlying skin was excised if on sonographic assessment, tumour-skin distance was $<13 \mathrm{~mm}$, to achieve adequate oncological clearance while maintaining the cutaneous viability.

\section{RESULTS}

Overlying skin was excised in all the study patients (mean tumour-skin distance: $5.32 \mathrm{~mm}$ ). None of the control group patients had undergone skin excision. In the study group, $86.6 \%$ of patients underwent flap reconstructions as compared to $46.6 \%$ in the control group. The deep margins were adequate in $85.7 \%$ of the study group patients and in $13.3 \%$ of the control group patients ( $\mathrm{p}$ $<0.001)$. All patients in the control group required adjuvant therapy due to adverse histopathological factors. During the follow up period of 2 years, the disease-free survival for both groups was comparable (log-rank test; $p=0.838$ )

\section{CONCLUSIONS}

The deep margin clearance is significantly improved by sonographic assessment as compared to the clinical judgment of skin involvement. Skin resection adds to the magnitude of surgery, but fewer patients will need receiving of adjuvant radiotherapy. The benefit on locoregional recurrence will require a study with larger numbers.

HOW TO CITE THIS ARTICLE: Thomas CT, Mukhopadhyay S, Irodi A, et al. Preoperative ultrasonographic determination of deep surgical margin in squamous cell carcinoma of buccal mucosa. J. Evolution Med. Dent. Sci. 2019;8(18):1422-1427, DOI: $10.14260 /$ jemds/2019/317

\section{BACKGROUND}

Oral cancer is the $3^{\text {rd }}$ most common cancer affecting Indians.[1] While in the western population buccal mucosa is involved in $10 \%$ of oral cancer patients, this figure is at $36 \%$ in the Indian population. ${ }^{2]}$ The higher proportion of buccal involvement is related to the widespread practice of chewing tobacco and placement of quid in the gingivobuccal sulcus. [3]

'Financial or Other Competing Interest': None.

Submission 07-04-2019, Peer Review 27-04-2019,

Acceptance 29-04-2019, Published 06-05-2019.

Corresponding Author:

Pranay Gaikwad,

Professor of Surgery and HOD,

Department of General Surgery Unit 1-

Head and Neck Oncosurgery,

Christian Medical College,

Vellore-632004, Tamilnadu, India.

E-mail: pranay@cmvellore.ac.in

DOI: $10.14260 /$ jemds/2019/317
The failure rates of treatment are high with locoregional recurrence rates between $40-80 \% .{ }^{[4]}$ While tumour biology may dictate some of these, a surgical resection with attention to margins in three dimensions should reduce this risk. The deep resection margin in a carcinoma buccal mucosa often falls short of the clinically uninvolved cheek skin without consideration of the depth of invasion of the tumour. This may account for close margins in the depth.

The incidence of close and positive margins varies between $25.5 \%$ and $59 \% .{ }^{[5-8]}$ It is not clear whether the margin status reported in literature includes deep margin along with mucosal or whether mucosal margins alone have been considered. This may have a role in the high local recurrence rates quoted for buccal carcinomas. Diaz et al quoted an incidence of invasion of buccinator of $34 \%$. The locoregional recurrence rates were $46 \%$ and $41 \%$ for patients with and without muscle invasion, respectively. But there was no mention of the deep margin status. [9] 
Ota et al proposed an anatomical classification of buccal mucosal carcinoma based on depth of invasion on ultrasonography. They resected skin only when the tumour breached the buccinator muscle into the subcutaneous tissues and thus achieved a disease-specific survival rate of $73.7 \%$ and a local control rate of $89.5 \% .[10]$ Liao et al reviewed their series of 331 patients with buccal carcinomas. All patients had a CT imaging to assess the distance of the skin from the tumour and if this was $\geq 13 \mathrm{~mm}$, they would preserve the skin. This was considering the $10 \mathrm{~mm}$ surgical clearance and a minimum of $3 \mathrm{~mm}$ of skin to be retained to maintain its vascularity. They had a 5 -year local control rate of $88 \%$. Their deep margin was positive in $<1 \%$ patients.[11]

We have conducted a study in our institute combining ultrasound evaluation of depth and surgical management based on a surgical margin rather than an anatomical margin.

\section{METHODS}

The study was designed as a prospective study of all patients with carcinoma of the buccal mucosa. For purposes of comparison a retrospective cohort was analysed.

A prior Institutional Review Board (IRB) approval and institutional funding for the study was obtained (Ref:FG/8804/03/2014). All patients were counselled and explained the details of the study and informed written consent was obtained prior to inclusion in the study.

The prospective group (study group) comprised of all patients with carcinoma of the buccal mucosa attending the Out-Patient department (OPD) between March 2014 and June 2015. All patients with newly diagnosed, resectable squamous cell carcinoma limited to the buccal mucosa were included and those with obvious skin involvement (skin fixity, peau d'orange and ulceration) were excluded.

The preoperative investigations were directed towards staging and assessment of fitness for surgery. Clinical staging was done by direct examination of the lesion and crosssectional imaging if the tumour was close to bone or retromolar trigone or if doubtful nodes were present. Chest $\mathrm{X}$ ray was done to rule out obvious lung secondaries.

All patients in the study group had a percutaneous ultrasound done to assess the maximum thickness of the lesion (A) and the minimum distance of the deepest aspect of the tumour from the skin (B). If the distance B was $\geq 13 \mathrm{~mm}$ then the overlying skin was preserved, and the line of excision passed through the subcutaneous tissue (Fig. 1) (At least $1 \mathrm{~cm}$ margin is needed for oncological clearance with an additional $3 \mathrm{~mm}$ of remaining skin to preserve subdermal vessels and ensure viability of overlying skin). If the distance ' $B$ ' was less than $13 \mathrm{~mm}$, then the line of excision passed through the skin, thus removing it en bloc with the carcinoma (Fig. 2)

The specimen was then oriented with markers on the mucosal aspect and on the deep aspect of the lesion and sent for routine histopathological examination.

We had 45 patients of buccal carcinoma in the period from Jan. 2008 to Dec. 2010 as part of a retrospective data that we had collected. Out of these 15 patients (Retrospective group) fulfilled the inclusion and exclusion criteria (i.e. there was no skin involvement on physical examination) that was formulated for the present study and these alone were, also, included as a comparable group. All patients were followed up for a period of 24 months.

\section{Statistical Analysis}

The distribution of tumour stage, surgical procedures, histopathological factors, adjuvant therapy in the prospective and retrospective groups were compared using Chi-square and Fisher's exact test. The Kaplan-Meier estimator was used to compare the disease-free survival in the two groups.

\section{RESULTS}

The male to female ratio in the study group was $2: 1$ and the male preponderance was seen in our retrospective group also $(93.3 \%)$. The mean age of the study group was 60.6 yrs and 52.3 yrs in the retrospective series.

\section{Surgery}

All study group patients required skin excision for clearance of tumour as the distance of tumour to skin was $<13 \mathrm{~mm}$. There was no case of skin excision in retrospective group as it was the practice to preserve skin with no clinical signs of involvement. There were significantly more patients who required flap reconstructions in study group than the retrospective group (Table 1 ).

\section{Histopathology}

In the study group, one patient had no tumour in the final post-surgical biopsy. He was staged as pT1 as the initial biopsy had shown early invasive squamous cell carcinoma. The pathological tumour and nodal staging were comparable between both groups (Table 2).

\section{Histopathological Margin Status}

In the study group, the mean distance of tumour from the skin (B, Fig. 1) as measured with ultrasound was $5.3 \pm 2.5$ $\mathrm{mm}$. All patients had less than $13 \mathrm{~mm}$ distance of tumour from the skin and thus required excision of skin. However, in the study group, one patient did not have any tumour in the postsurgical biopsy specimen and hence deep margin status was assessed for $14 / 15$ (93.3\%) patients.

The deep margins were adequate $(>5 \mathrm{~mm})$ in $12 / 14$ $(85.7 \%)$ patients and this was a significant improvement over the retrospective group where negative margins were achieved in $13.3 \%(\mathrm{p}<0.001)$

In the study group, the mucosal margin was negative in $10 / 14(71.4 \%)$ and close in $4 / 14(28.5 \%)$ patients; and negative in $3 / 15$ (20\%), close in $8 / 15$ (53.3\%) and positive in $4 / 15(26.6 \%)$ patients in the retrospective group. For the statistical analysis, the close and positive margins were combined as inadequate margins and the difference between the two groups was statistically significant (Fisher's exact test, $\mathrm{p}=0.004$ ). (Table 2)

\section{Adjuvant Therapy}

Six patients in the study required adjuvant radiotherapy for adverse histopathological factors; one required adjuvant chemotherapy in addition to radiotherapy. Four patients completed radiotherapy while two defaulted without taking a single dose of radiotherapy and one defaulted on the second dose of chemotherapy in the study group (Table 3). Significantly more patients required adjuvant radiotherapy and chemotherapy in the retrospective group. 


\section{Locoregional Recurrences}

There were three patients with recurrences in the study group, two local buccal recurrence and one nodal recurrence. For purposes of analysis, we looked at locoregional recurrence as a composite outcome variable. Univariate analysis in the study group showed that factors like tumour grade, pathological tumour stage, pathological nodal stage, perineural invasion (PNI), extranodal spread (ENS), mucosal margin status and deep margin status were not statistically significant predictors of locoregional recurrence. (Table 4)

Univariate analysis in the retrospective group showed that prognostic factors like tumour grade, pathological tumour stage, pathological nodal stage, mucosal margin status and deep margin status were not statistically significant predictors of locoregional recurrence. (Table 4)

\section{Follow Up}

All the patients were followed up for at least 2 years or till an event occurred. The mean follow-up period was 20 months in the study group and 28.3 months in the retrospective group. Kaplan Meier survival curves comparing both groups for loco-regional recurrence were plotted and the groups did not differ significantly (Fig. 3; log rank test; $\mathrm{p}=0.838$ ).

\begin{tabular}{|c|c|c|c|}
\hline & Study Group & Retrospective Group & p Value (Fisher's Exact Test) \\
\hline Skin excision required & $15(100 \%)$ & 0 & $<0.0001$ \\
\hline \multicolumn{2}{|c|}{ Reconstruction } \\
\hline None & $2(13.3 \%)$ & $8(53.3 \%)$ & 0.025 (comparing no flap vs. any \\
flap)
\end{tabular}

\begin{tabular}{|c|c|c|c|}
\hline & Study Group & Retrospective Group & p Value (Chi-Square test) \\
\hline Tumour staging & & & \multirow[t]{3}{*}{1} \\
\hline pT1-T2 & $13(86.6 \%)$ & $12(80 \%)$ & \\
\hline pT3-T4 & $2(13.3 \%)$ & $3(20 \%)$ & \\
\hline Nodal staging & & & \multirow{3}{*}{0.385} \\
\hline $\mathrm{pN}+$ & $2(14.3 \%)$ & $4(30.8 \%)$ & \\
\hline pN0 & $12(85.7 \%)$ & $9(69.2 \%)$ & \\
\hline Deep margin stat & & & \multirow{4}{*}{$<0.001$} \\
\hline Negative & $12(85.7 \%)$ & $2(13.3 \%)$ & \\
\hline Close & $2(14.3 \%)$ & $8(53.3 \%)$ & \\
\hline Positive & 0 & $5(33.3 \%)$ & \\
\hline \multicolumn{4}{|c|}{ Mucosal Margin Status } \\
\hline Negative & $10(71.4 \%)$ & $3(20 \%)$ & \multirow{3}{*}{0.009} \\
\hline Close & $4(28.6 \%)$ & $8(53.3 \%)$ & \\
\hline Positive & 0 & $4(26.6 \%)$ & \\
\hline \multicolumn{4}{|c|}{ Table 2. Distribution of Histopathological Variables Between the Study and Retrospective Groups } \\
\hline
\end{tabular}

\begin{tabular}{|c|c|c|c|}
\hline & Study Group & Retrospective Group & p Value (Fisher's Exact Test) \\
\hline Adjuvant RT indicated & $6(40 \%)$ & $15(100 \%)$ & 0.001 \\
\hline Adjuvant RT completed & $4(26.6 \%)$ & $9(60 \%)$ & 1.00 \\
\hline Adjuvant chemotherapy indicated & $1(6.6 \%)$ & $8(53.3 \%)$ & 0.014 \\
\hline Adjuvant chemotherapy completed & 0 & $1(6.6 \%)$ & 1.00 \\
\hline \multicolumn{2}{|r}{ Table 3. Distribution of Adjuvant Therapy in Study and Retrospective Groups } \\
\hline
\end{tabular}

\begin{tabular}{|c|c|c|c|c|c|c|}
\hline \multirow[b]{2}{*}{ Risk Factors } & \multicolumn{3}{|c|}{ Study Group } & \multicolumn{3}{|c|}{ Retrospective Group } \\
\hline & No Recurrence & Recurrence & p Value & No Recurrence & Recurrence & p Value \\
\hline \multicolumn{7}{|c|}{ Tumour Grade } \\
\hline Well differentiated & 9 & 1 & & 5 & 0 & \\
\hline Moderately differentiated & 2 & 2 & 0.176 & 7 & 3 & 0.505 \\
\hline \multicolumn{7}{|c|}{ pT } \\
\hline T1-2 & 10 & 3 & & 9 & 3 & \\
\hline T3-4 & 2 & 0 & 1.0 & 3 & 0 & 1.0 \\
\hline \multicolumn{7}{|c|}{ pN } \\
\hline pN0 & 9 & 3 & & 9 & 0 & \\
\hline $\mathrm{pN}+$ & 2 & 0 & 1.0 & 2 & 2 & 0.077 \\
\hline \multicolumn{7}{|c|}{ PNI } \\
\hline Yes & 1 & 0 & & & & \\
\hline No & 10 & 3 & 1.0 & & & \\
\hline \multicolumn{7}{|c|}{ ENS } \\
\hline Yes & 1 & 0 & & & & \\
\hline No & 10 & 3 & 1.0 & & & \\
\hline
\end{tabular}




\begin{tabular}{|c|c|c|c|c|c|c|}
\hline \multicolumn{7}{|c|}{ Mucosal Margin Status } \\
\hline Adequate & 9 & 1 & 3 & 0 & 3 \\
\hline Inadequate & 2 & 2 & 0.176 & 9 & 1.0 \\
\hline \multicolumn{7}{|c|}{ Deep Margin Status } \\
\hline Adequate & 10 & 2 & & 1 & 1 & \\
\hline Inadequate & 1 & 1 & 0.396 & 11 & 2 & 0.371 \\
\hline
\end{tabular}
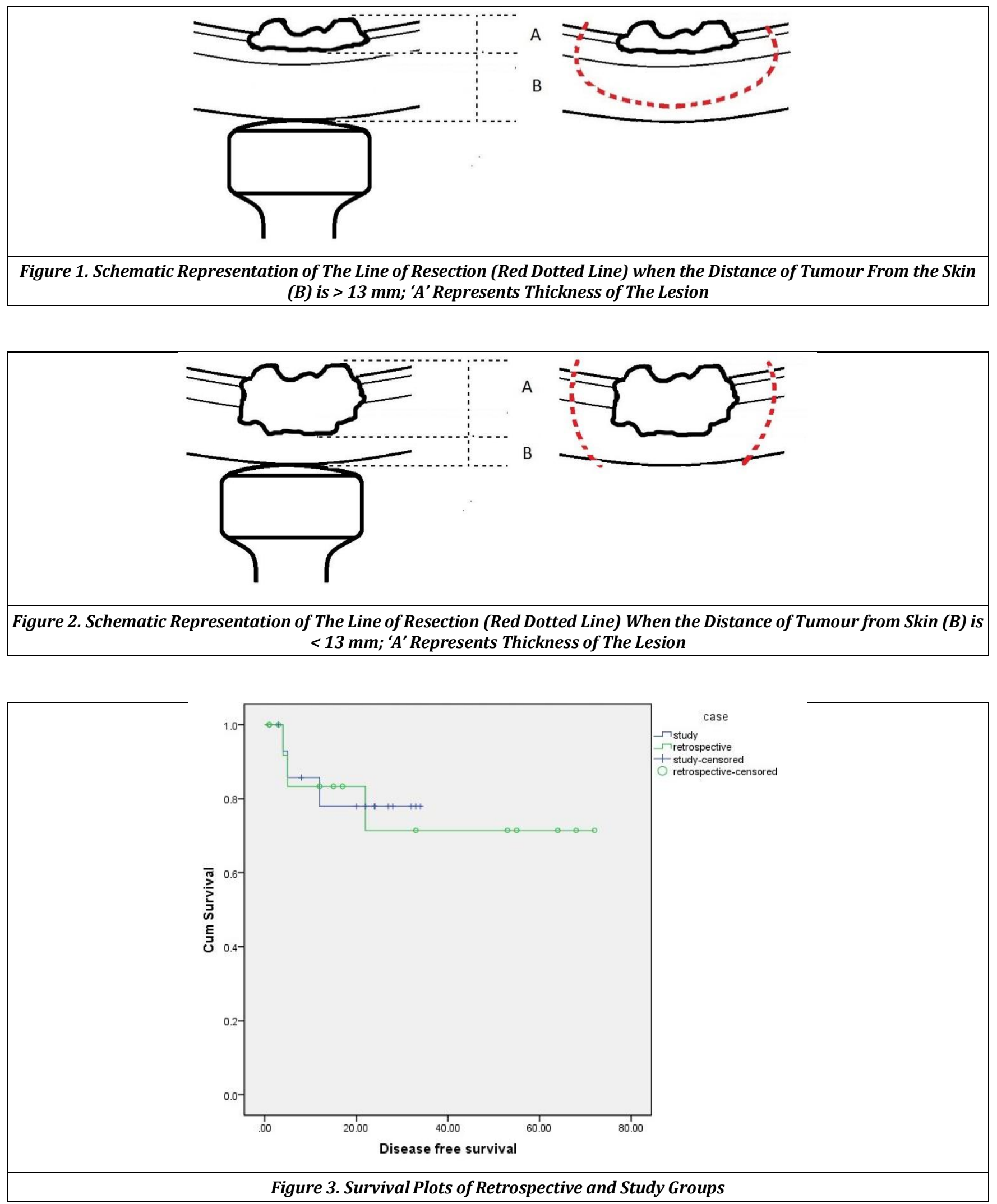


\section{DISCUSSION}

The male to female ratio in our study was 2:1 and the male preponderance was seen in our retrospective series also. The mean age was 60.6 yrs. This differed significantly with our patients in 2008-2010 by just under a decade. In other series in Asian countries the age tended to be in the $6^{\text {th }}$ decade while in the Western world buccal cancer was seen as an affliction of the $7^{\text {th }}$ to $8^{\text {th }}$ decade. $[12,13]$

The distribution of the pathological tumour and nodal stages was not significantly different in the study and retrospective groups. The prevalence of lymphovascular invasion, perineural invasion $(1 / 15)$ and extranodal spread $(1 / 15)$ was low in our study group. Most of the tumours in the study group were well differentiated (10/14, 71.4\%). Fang et al reported that $95 \%$ of these tumours were well differentiated.[14] One patient did not have residual tumour in the postoperative specimen and was reported as early invasive in the preoperative biopsy.

In our study group, the mean distance of the tumour from the skin was $5.3 \mathrm{~mm}$, as measured with ultrasound, with none having a minimum safe distance of $13 \mathrm{~mm}$. This implied that an oncologic ally safe resection with preservation of skin could not be done, just based on clinical judgement, in any of our patients. In Liao et al study, 69.5\% patients underwent excision of skin based on radiological and clinical criteria.[11] Deconde et al reported a rate of skin resection in $29.1 \%$ of patients with buccal carcinoma, half of whom had T4 tumours.[15] Clearly, Liao et al has shown better loco-regional control rates following treatment based on evaluation of skin involvement by radiological criteria. This is objective and reproducible as against the previous clinical criteria. But, a higher percentage of patients have had skin excision along with the tumour, as is seen in our series also.

The deep margin status was negative in $85.7 \%$ of patients, $2 / 14(14.3 \%)$ had close margins. There were no cases of positive deep margins. This was a statistically significant improvement from our retrospective series in which $86.6 \%$ of patients had either close or positive margins. On univariate analysis, thickness of tumour, pathological tumour staging, and pathological nodal staging had no demonstrable effect on the deep margin status. There is no formal reporting of deep margin status in the literature apart from Liao et al, who was able to achieve $99 \%$ adequate deep margins with his method of using radiological criteria and radical excision of skin. It is worth reporting this in future studies and analysing the impact on loco-regional control.

We had better control of mucosal margins in the study group with $71.4 \%$ having adequate margins against $22.2 \%$ in the retrospective group. Since 2013, we have adopted a 1.5 $\mathrm{cm}$ margin of excision against a previous $1 \mathrm{~cm}$ margin for the mucosal resections. That aside, the use of ultrasound would have contributed little to these figures. This improvement in the mucosal margin status will have a bearing on locoregional control and how much can be attributed to deep margin status will have to be seen.

All our patients required some form of closure or cover of the external defect resulting from concomitant skin resection. This reiterates the need for selection of robust flap cover, factoring the increased cost and operative time into initial planning and counselling of patients. The retrospective group had fewer flap reconstructions but significantly more patients requiring adjuvant radiotherapy due to inadequate margins.
This would also add to cost and duration of the entire therapy.

The mean follow-up of the study group was 20 months and both groups were analysed for disease free survival. There was no significant difference in both groups. This could be attributed to the fact that the sample size, though adequate to detect an improvement in the deep resection margin, was not powered to detect differences in locoregional recurrences. Another contributing factor may be the higher rate of adjuvant radiotherapy in the retrospective group of patients.

\section{CONCLUSIONS}

Ultrasound can measure of the distance of the tumour from the skin and this can guide in deciding on skin resection. Deep margin clearance is significantly improved by this approach when compared to the erstwhile practice of judging skin involvement clinically. Skin resection adds to the magnitude of the surgery and the cost in the form of additional procedures for flap cover but leads to lesser patients receiving adjuvant radiotherapy. The benefit of locoregional recurrence will require a study with larger numbers.

\section{REFERENCES}

[1] Bray F, Ren JS, Masuyer E, et al. Global estimates of cancer prevalence for 27 sites in the adult population in 2008. Int J Cancer 2013;132(5):1133-45.

[2] Yeole BB, Ramanakumar AV, Sankaranarayanan R. Survival from oral cancer in Mumbai (Bombay), India. Cancer Causes Control 2003;14(10):945-52.

[3] Iype EM, Pandey M, Mathew A, et al. Squamous cell cancer of the buccal mucosa in young adults. Br J Oral Maxillofac Surg 2004;42(3):185-9.

[4] Liao CT, Wang HM, Ng SH, et al. Good tumour control and survivals of squamous cell carcinoma of buccal mucosa treated with radical surgery with or without neck dissection in Taiwan. Oral Oncol 2006;42(8):8009.

[5] Loree TR, Strong EW. Significance of positive margins in oral cavity squamous carcinoma. Am J Surg 1990;160(4):410-4.

[6] Shaw RJ, McGlashan G, Woolgar JA, et al. Prognostic importance of site in squamous cell carcinoma of the buccal mucosa. $\mathrm{Br} \mathrm{J}$ Oral Maxillofac Surg 2009;47(5):356-9.

[7] Priya SR, D'Cruz AK, Pai PS. Cut margins and disease control in oral cancers. J Cancer Res Ther 2012;8(1):74-9.

[8] Lin CS, Jen YM, Kao WY, et al. Improved outcomes in buccal squamous cell carcinoma. Head Neck 2013;35(1):65-71.

[9] Diaz EM, Holsinger FC, Zuniga ER, et al. Squamous cell carcinoma of the buccal mucosa: one institution's experience with 119 previously untreated patients. Head Neck 2003;25(4):267-73.

[10] Ota Y, Aoki T, Karakida K, et al. Determination of deep surgical margin based on anatomical architecture for local control of squamous cell carcinoma of the buccal mucosa. Oral Oncol 2009;45(7):605-9. 
[11] Liao CT, Huang SF, Chen IH, et al. When does skin excision allow the achievement of an adequate local control rate in patients with squamous cell carcinoma involving the buccal mucosa? Ann Surg Oncol 2008;15(8):2187-94.

[12] Huang CH, Chu ST, Ger LP, et al. Clinicopathologic evaluation of prognostic factors for squamous cell carcinoma of the buccal mucosa. J Chin Med Assoc 2007;70(4):164-70.

[13] Ghoshal S, Mallick I, Panda N, et al. Carcinoma of the buccal mucosa: analysis of clinical presentation, outcome and prognostic factors. Oral Oncol 2006;42(5):533-9.
[14] Fang FM, Leung SW, Huang CC, et al. Combinedmodality therapy for squamous carcinoma of the buccal mucosa: treatment results and prognostic factors. Head Neck 1997;19(6):506-12.

[15] DeConde A, Miller ME, Palla B, et al. Squamous cell carcinoma of buccal mucosa: a 40-year review. Am J Otolaryngol 2012;33(6):673-7. 